Gazi University
Journal of Science
http://dergipark.gov.tr/gujs

\title{
Dimeric aza-BODIPY and Dichloro-aza-BODIPY: A DFT Study
}

\author{
Mehmet Emin CINAR* \\ Universität Siegen, Department Chemie-Biologie, Adolf-Reichwein-Str. 2, 57068 Siegen, Germany
}

Highlights

- DFT computations were performed on dimeric aza-BODIPY molecule and its chlorinated form.

- Their intriguing photophysical behaviors were investigated by conducting TDA-DFT calculations.

- The results were compared to shed light on the effect of chlorination.

\begin{tabular}{l} 
Article Info \\
\hline Received: 24 Dec 2020 \\
Accepted: 04 June 2021 \\
Keywords \\
\hline Dimeric aza-BODIPY \\
Computation \\
(TDA)-DFT \\
NBO Analysis \\
Optoelectronic property
\end{tabular}

\section{INTRODUCTION}

The complexation of dipyrromethene with $\mathrm{BF}_{3}$ leads to the formation of boron dipyrromethene $(4,4-$ difluoro-4-bora-3a,4a-diaza-s-indacen) also known as BODIPY (

Figure 1) [1,2]. The effect of substitution pattern especially meso-substitution on spectroscopic and photophysical properties of BODIPY has been meticulously explored [3,4]. Moreover, BODIPY dimers linked via $\alpha$ or $\beta$ positions of monomer units have also been investigated thoroughly experimentally and computationally [5-7]. Replacement of carbon at the meso position of BODIPY by nitrogen renders azaBODIPY $[8,9]$. Although aza-dipyrromethene has been known since the $1940 \mathrm{~s}$, its $\mathrm{BF}_{2}$ chelated form (azaBODIPY) was firstly achieved by Boyer et. al in 1993 [10, 11]. Aza-BODIPY has also been extensively studied by considering its fused aromatic systems and substitution effects on its chemical and photophysical properties [12,13]. Absorption maxima of aza-BODIPY are shifted to the near infrared region compared to BODIPY emerging from the diminishing of a gap between the highest occupied molecular orbital (HOMO) - the lowest unoccupied molecular orbital (LUMO) [14]. Both electron donating groups at 3/5-positions and electron withdrawing units at 1/7-positions decrease the HOMO-LUMO gaps, and hence result in bathochromic shifts. Moreover, it is reported that they have sharp absorption peaks with extinction coefficients between 75,000 and $85,000 \mathrm{M}^{-1} \mathrm{~cm}^{-1}$ [10]. The intriguing photophysical properties of azaBODIPYs allow aza-BODIPYs to be utilized as key materials in the construction of solar cells, logic gates, and agents for biolabeling, boron neutron capture therapy (BNCT) and Photodynamic therapy (PDT) [15-17]. Homocoupling of aza-BODIPY through $\beta$-position gives rise to the formation of dimeric azaBODIPY. Despite the fascinating effect of dimerization on the optoelectronic properties that is, enhancing

*e-mail:emin.cinar@uni-siegen.de 
photophysical properties, shifting the absorption maxima to the longer wavelengths, a limited number of studies involving dimeric aza-BODIPY were reported $[18,19]$.

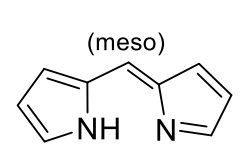

Dipyrromethene

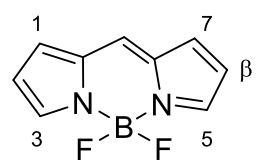

BODIPY

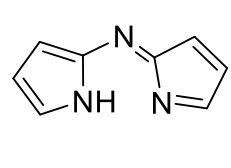

aza-Dipyrromethene

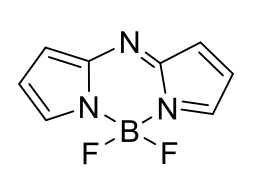

aza-BODIPY

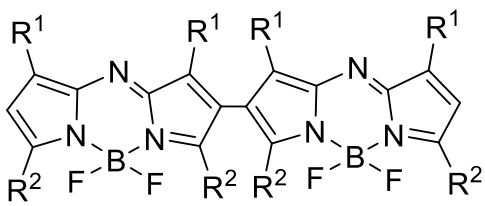

Dimeric aza-BODIPY

\section{Figure 1. (aza)-BODIPY, dimeric aza-BODIPY and their precursor compounds; dipyrromethene and aza-dipyrromethene}

Bard and co-workers [19] investigated the photophysical and electrochemical properties of dimeric azaBODIPY $\left(\mathrm{R}^{1}=\mathrm{R}^{2}=\mathrm{Ph}\right)$, which was synthesized from tetra-phenyl substituted monomer via oxidative homocoupling. It was pointed out that the enhanced electronic interactions and bathochromic shift to nearinfrared region endow this dye molecule with a wide applicability. Moreover, Gul [20] investigated the effect of $\mathrm{Cl}$ atom located at the para-positions of the distal phenyl groups on the properties of dimeric azaBODIPY $\left(\mathrm{R}^{1}=p\right.$-ClPh, $\left.\mathrm{R}^{2}=\mathrm{Ph}\right)$ indicating a significant bathochromic shift of $57 \mathrm{~nm}$ and a large Stokes shift of $34 \mathrm{~nm}$ compared to its monomer. In this study, DFT computations were performed to shed light on the electronic structures and Frontier Molecular Orbitals (FMOs) of the dimeric aza-BODIPY molecule and its chlorinated form. Tamm-Dancoff approximation (TDA)-DFT [21,22] calculations were conducted to unveil their optoelectronic properties.

\section{MATERIAL METHOD}

Geometry optimizations of aza-BODIPY dimers were performed in a vacuum at DFT level using the Gaussian 09 package program [23] Meta hybrid M06-2X [24] functional was employed with Duning's correlation-consistent double-zeta basis set cc-pVDZ due to its good results in the literature for geometry optimizations of the dimeric aza-BODIPY dimer and also metal complexes [25,26]. It was reported that solvent effect on geometry optimization of BODIPY is insignificant and the basis set affects moderately. Therefore, the global minima of the dimers were predicted by performing calculations at M06-2X/cc-pVDZ level of theory in the gas phase. The atom-pairwise dispersion correction (D3) [27,28] was included for M06-2X functional with the help of the keyword $\operatorname{IOp}(3 / 124=30)$ [29]. As the predicted results obtained utilizing the M06-2X functional are strongly dependent on the choice of grid and "ultrafine" grid (a pruned grid of 99 radial shells and 590 angular points per shell) is suitable for optimizations of larger molecules with many soft modes, an "Ultrafine" grid was considered for numerical integration [30,31]. Convergence of a maximum force $=1.2 \times 10^{-5}$ a.u. and RMS force $=2.0 \times 10^{-6}$ a.u. were achieved for the ground-state optimizations. The minima of the calculated structures were verified by analyzing the harmonic vibrational frequencies using analytical second derivatives, which have NIMAG $=0$. Orbital composition analysis was performed using the Multiwfn program (a multifunctional wavefunction analyzer) with Mulliken partition [32]. Natural Bond Orbital (NBO) analyses [33-35] were realized with NBO 3.1 [36,37] linked through Gaussian 09 using cc-pVTZ [23] basis set to shed light on the electronic structures and the second-order perturbative estimates of bond-antibond interactions. Single point (SP) calculations were performed on gas phase optimized geometries at UM062X/cc-pVDZ//M06-2X/cc-pVDZ level of theory to predict the first vertical ionization energies (VIE). The time-dependent DFT (TD-DFT) calculations were performed with TDA and frozen core option based on the reports which indicate that excitation energies obtained from TDA computations are very similar to those predicted using the full time-dependent (TD) method. Moreover, TDA demonstrated better performances in the estimations involving purely valence excitations, transition metals and, also large conjugated systems [38]. TDA-DFT computations were performed using TDA keyword provided in the Gaussian 09 package and the PBE0 [39] functional owing to its good performance in the prediction of vertical excitation energies yielding the least amounts of mean absolute deviation of $0.267 \mathrm{eV}$ [25]. The same basis set was utilized for the gas phase calculations of the lowest singlet-singlet vertical excitations because of the use of larger basis sets (aug-cc-pVDZ, cc-pVTZ, aug-cc- 
pVTZ) and density fitting basis sets (def2-TZVPP, def2-QZVPP) has little effect on the excitation energies and the solvent effect on the excited-state properties of this type of molecules was observed to be negligible [40,41]. Although TDA computations both in gas phase and in tetrahydrofuran (THF) provided hypsochromic deviations compared to the experimentally recorded value for dichloro-aza-BODIPY, the deviation is smaller in THF $(38 \mathrm{~nm})$ than in vacuum $(54 \mathrm{~nm})$. Moreover, the larger oscillator strength $(F=0.906)$ was predicted in solvent with respect to that in gas phase $(F=0.588)$. Therefore, incorporation of the solvent effects was realized by using self-consistent reaction field (SCRF) with the integral equation formalism of the Polarizable Continuum Model (IEFPCM) [42]. IEFPCM is a widely used methodology in the computational chemistry, which is the default SCRF method in Gaussian 09 program package. IEF is less affected by both the numerical and physical errors on the apparent charges compared to conventional PCM method and it is among the most effective methods for standard isotropic dielectrics. THF $(\varepsilon=7.4257)$ was chosen as solvent to mimic the UV-Vis measurement conditions. To obtain absorption bands, the calculations were performed for singlets with the number of states (NStates) of 50. The visualization of MOs with isosurface value of 0.03 a.u. was performed with GaussView 5.0 [43]. IR and absorption spectra were generated by using GaussSum 3.0 [44].

\section{RESULTS AND DISCUSSION}

\subsection{Geometry Optimization}

X-ray crystal structure of dichloro-substituted 2,6-dibromo-aza-BODIPY compound was considered as a starting point [20]. Bromine atoms were replaced by $\mathrm{H}$ atoms and $\beta \mathrm{C}-\mathrm{Br}$ distances in crystal structure were arranged to $1.08509 \AA$ based on the $\beta \mathrm{C}-\mathrm{H}$ distances of dimeric aza-BODIPY molecule provided in the literature [25]. Aza-BODIPY dimer was constructed by connecting two $\beta C$ of monomeric units and keeping the distance of $1.45560 \AA$. Dihedral angle around $\beta C-\beta C$ bond was set to $40^{\circ}$ to avoid the bulkiness of aryl units, but at the same time to maintain the conjugation as high as possible. It is known that the dihedral angle of above $30^{\circ}$ results in diminishing electron conjugation in the $\pi$-backbone and hence altering the optoelectronic properties of target materials [45]. Crystal structure also clearly depicts a nearly $\sigma_{\mathrm{v}}$ symmetry, which is slightly destroyed by an intersect angle of $4.7^{\circ}$ between two pyrrole rings [20]. However, the close $\sigma_{\mathrm{v}}$ symmetry observed in a monomer disappears in its dimers. C2 symmetry was employed with the keyword $\mathrm{Symm}=(\mathrm{PG}=\mathrm{C} 2$, follow $)$ in the route section to accelerate the optimization calculations. The predicted Cartesian coordinates are provided in the Supporting Material (S1).

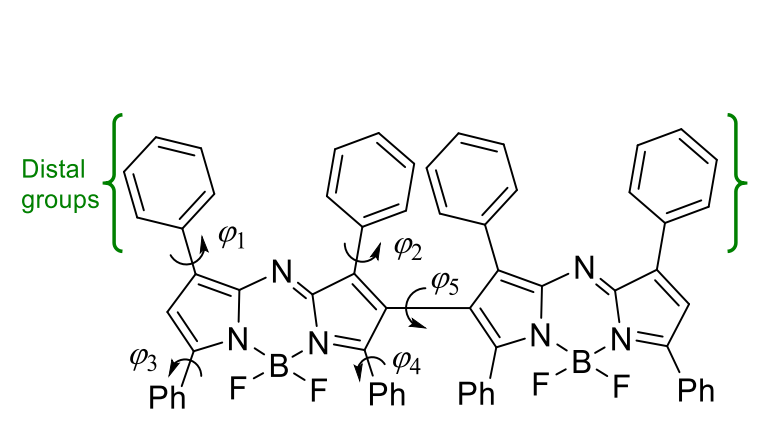

Dimeric aza-BODIPY

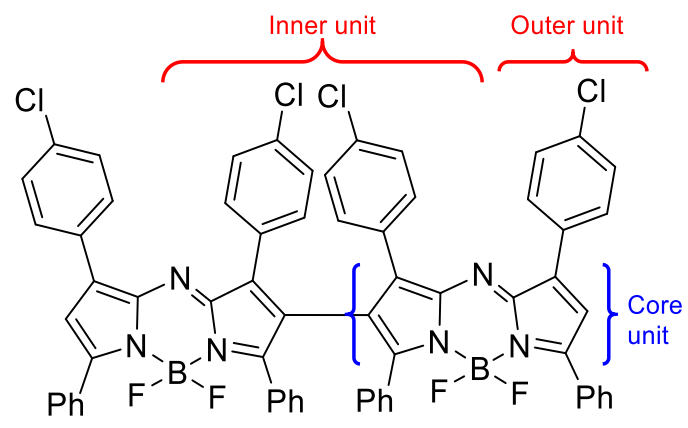

Dimeric dichloro-aza-BODIPY

Figure 2. Dimeric aza-BODIPY and dimeric dichloro-aza-BODIPY subjected to DFT computations

Gas phase DFT level optimization of dimeric aza-BODIPY and dimeric dichloro-aza-BODIPY compounds demonstrated that dimerization of monomeric aza-BODIPY and dichloro-aza-BODIPY cannot change the planarity of core units. The greatest deviation of $1.8^{\circ}$ was observed with the $\mathrm{N}$ atom of the pyrrole ring proximate to the dimer connection in both dimers, which brought about the intersection angle of $2.4^{\circ}$ and $2.5^{\circ}$ between two pyrrole rings in the aza-BODIPY units, respectively (

Figure 2). This prediction is well in alignment with the reported $\mathrm{X}$-ray crystal structure analysis of dichloroaza-BODIPY, in which the intersect angles of the pyrrole rings were recorded to be $4.1^{\circ}$ and $4.7^{\circ}[20,46]$. 
The distal dihedral angles $\varphi 1$ and $\varphi 2$ were estimated to be $25^{\circ}$ and $43^{\circ}$, respectively, which indicate the effect of dimerization on the torsion angle $\varphi 2$ emerging from the sterically bulky inner distal phenyl rings and their proper arrangements to form slipped-stack $\pi$ - $\pi$ interactions with a distance of $3.4 \AA$. Zhang et al [47] performed B3LYP/TZVP level optimization on aza-BODIPY providing $\varphi 1$ and $\varphi 2$ as $24^{\circ}$ and $30^{\circ}$ with a $\pi-\pi$ stacking in a distance of $3.4^{\circ}$, which noticeably points out the dimerization responsible for the large $\varphi 2$ of $43^{\circ}$. On the other hand, two bromine atoms at the $\beta$-positions of pyrrole units in the X-ray crystal structure give rise to the rotation of the distal phenyl groups in such a way that slipped-stack $\pi$ - $\pi$ interactions between aryl units are completely removed. Based on the X-ray crystal structure analysis, this phenomenon was explained by the crystal lattice containing $\mathrm{Cl}-\pi$ and $\mathrm{Br}-\pi$ stackings, which form a 2-dimensional supramolecular assembly in distances around $3.3 \AA$ [20]. Computations suggest the presence of nonbonding $\mathrm{CH} \cdot \bullet \mathrm{N}$ interactions in $2.38 \AA$ between ortho- $\mathrm{CH}$ of outer distal phenyl units and adjacent $\mathrm{N}$ (meso), which do not show up in inner distal phenyl groups owing most probably to the strong intermolecular $\pi-\pi$ interactions formed between inner distal aryl units [48]. $\mathrm{C}_{\mathrm{Ar}}-\mathrm{Cl}$ bond lengths were calculated to be $1.74 \AA$ which are somewhat longer than those in the crystal structure by $0.02 \AA$. Fluorine atoms sitting on the boron centers are also involved in non-covalent interactions with the ortho- $\mathrm{CH}$ of proximate phenyl units in 2.06 $\AA$, which decreases the dihedral angle between the outer phenyl and core unit to $30^{\circ}$. However, the strong $\pi-\pi$ interactions between inner phenyl units hinders the diminishing of torsional angles, hence gives rise to a large deviation of inner phenyl moieties from the planarity of the BODIPY unit with dihedral angles of up to $46^{\circ}$. Two fluorine atoms are separated from each other by $2.30 \AA$ via a boron atom with the angle of $112^{\circ}$, which was observed in the crystal structure as the distance of $2.24 \AA$ and the angle of $111^{\circ}$ indicating the well alignment of estimated geometric parameters with those belonging to X-ray crystal structure. The DFT level predictions provided the length of the dimeric linkages as $1.467 \AA$ and the dihedral angles $(\varphi 5)$ between two monomeric units to be $48^{\circ}$ in both dimers which clearly point out the lack of significant effects of chlorine atoms on the computed geometries. Rotation around this single bond by scanning $\varphi 5$ undoubtedly renders two maxima with different energies and four minima with two different energy levels indicating two enantiomeric pairs. The conformation of dimers reported in this work is well in alignment with the one provided in the literature [25].

\subsection{IR Spectra and Molecular Electrostatic Potential}

The optimized geometries and vibrational spectra of dimeric (dichloro)-aza-BODIPY compounds at M06$2 \mathrm{X} / \mathrm{cc}-\mathrm{pVDZ}$ level are given in

Figure 3. Their predicted vibrational spectra are matching well with the experimentally recorded spectra $[20,49]$. The estimated vibrations and their force constants (in mDyne/A) are listed for the dimeric azaBODIPY molecule as 3236 (w, 6.78, Ar-CH), 1608 (s, 14.9, C=C), 1594 (s, 13.0, C=C), 1557 (m, 5.98, $\mathrm{C}=\mathrm{C}), 1453(\mathrm{~m}, 5.81, \mathrm{~B}-\mathrm{N}), 1139(\mathrm{~m}, 1.44, \mathrm{~B}-\mathrm{F}), 1075 \mathrm{~cm}^{-1}$ (s, 1.89, C-H); and for the dichloro substituted one as $3236(\mathrm{w}, 6.78, \mathrm{Ar}-\mathrm{CH}), 1609(\mathrm{~s}, 14.7, \mathrm{C}=\mathrm{C}), 1591$ (s, 13.2, C=C), $1556(\mathrm{~m}, 6.25, \mathrm{C}=\mathrm{C}), 1455(\mathrm{~m}$, 6.19, B-N), 1141 (m, 1.30, B-F), 1074 (s, 2.34, C-H), $694 \mathrm{~cm}^{-1}$ (w, 1.78, C-Cl) (Supporting Material, S2 S12). As anticipated large force constants belong to double bonds, whereas small force constants are attributed to B-F and $\mathrm{C}-\mathrm{Cl}$ bonds.
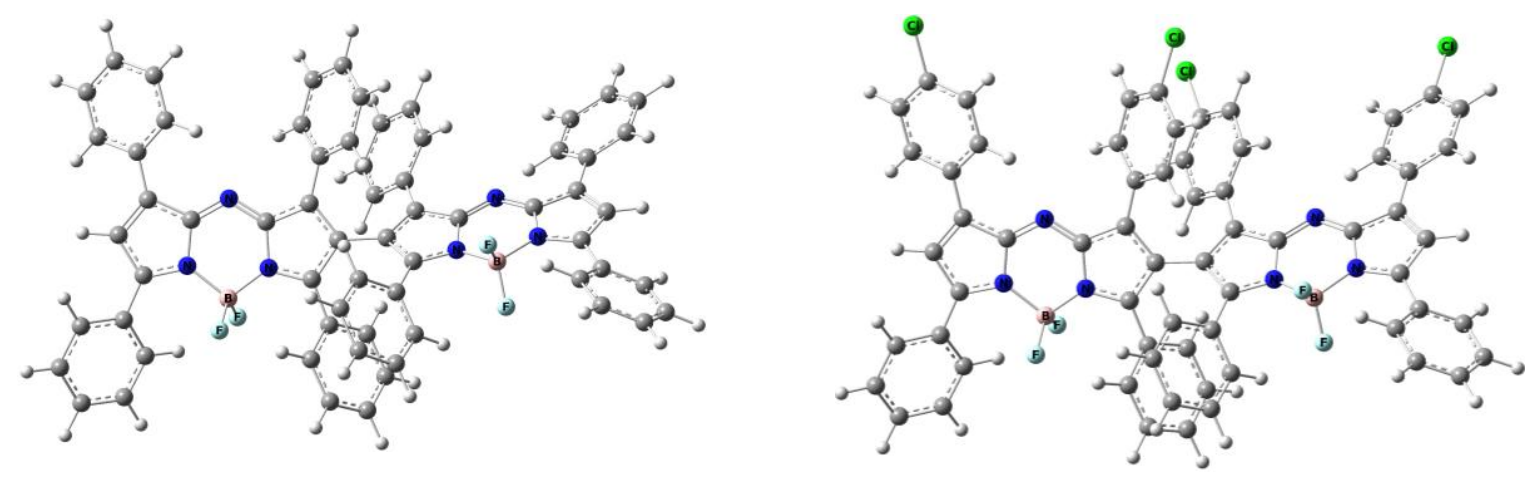

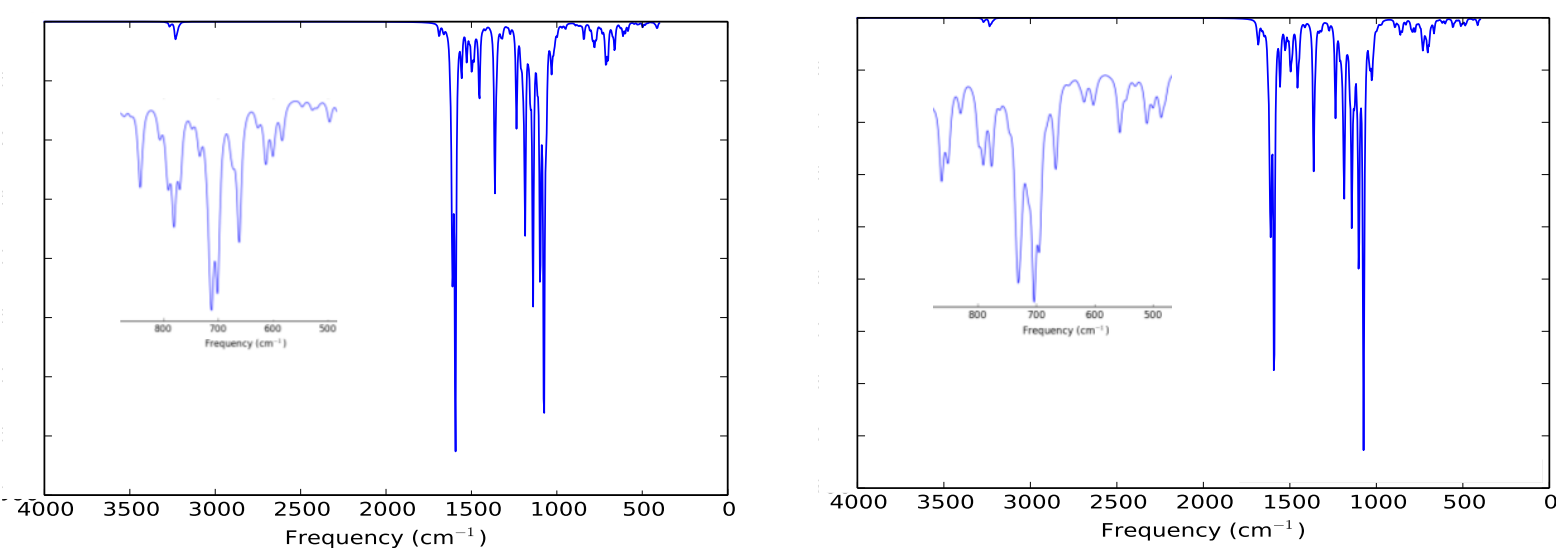

Figure 3. Optimized structures and vibrational spectra of dimeric aza-BODIPY (left) and dimeric dichloro-aza-BODIPY (right) compounds at M06-2X/cc-pVDZ level

Molecular Electrostatic Potential (MEP) maps demonstrate the charge distributions of compounds and provide information about the electron rich, region of attractive potential appearing in red, and electron deficient, region of repulsive potential appearing in blue, sites of the molecules [50-52]. MEP maps of dimeric aza-BODIPY and dimeric dichloro-aza-BODIPY compounds obtained at the same level of theory are depicted in

Figure 4. For both molecules, it was observed that negative charges are mostly located on the fluorine (0.21 a.u.) and nitrogen (-0.19 - -0.23 a.u.) atoms, and in dichloro-derivative slightly on the chlorine atoms (-0.05 a.u.). On the other hand, positive charges are mainly found on the carbon atoms, which are connected to nitrogen atoms, and largely on boron atoms (+0.26 a.u.). Another important molecular property is the dipole moment, which helps in differentiating between polar and non-polar compounds. The larger the dipole moment, the greater the polarity of a molecule [53,54]. The dipole moments of dimeric aza-BODIPY and dimeric dichloro-aza-BODIPY molecules were predicted to be 1.778 and 4.927 Debyes, respectively, with the almost opposite direction of dipole moment vectors provided in

Figure 4. The 2.8-fold difference between the dipole moments with quasi-opposite directions clearly emphasizes the substantial influence of chlorine atoms, whose positive mesomeric effect is more pronounced than its negative inductive effect, at the para positions of distal phenyl units on the polarities and charge distributions of molecules.

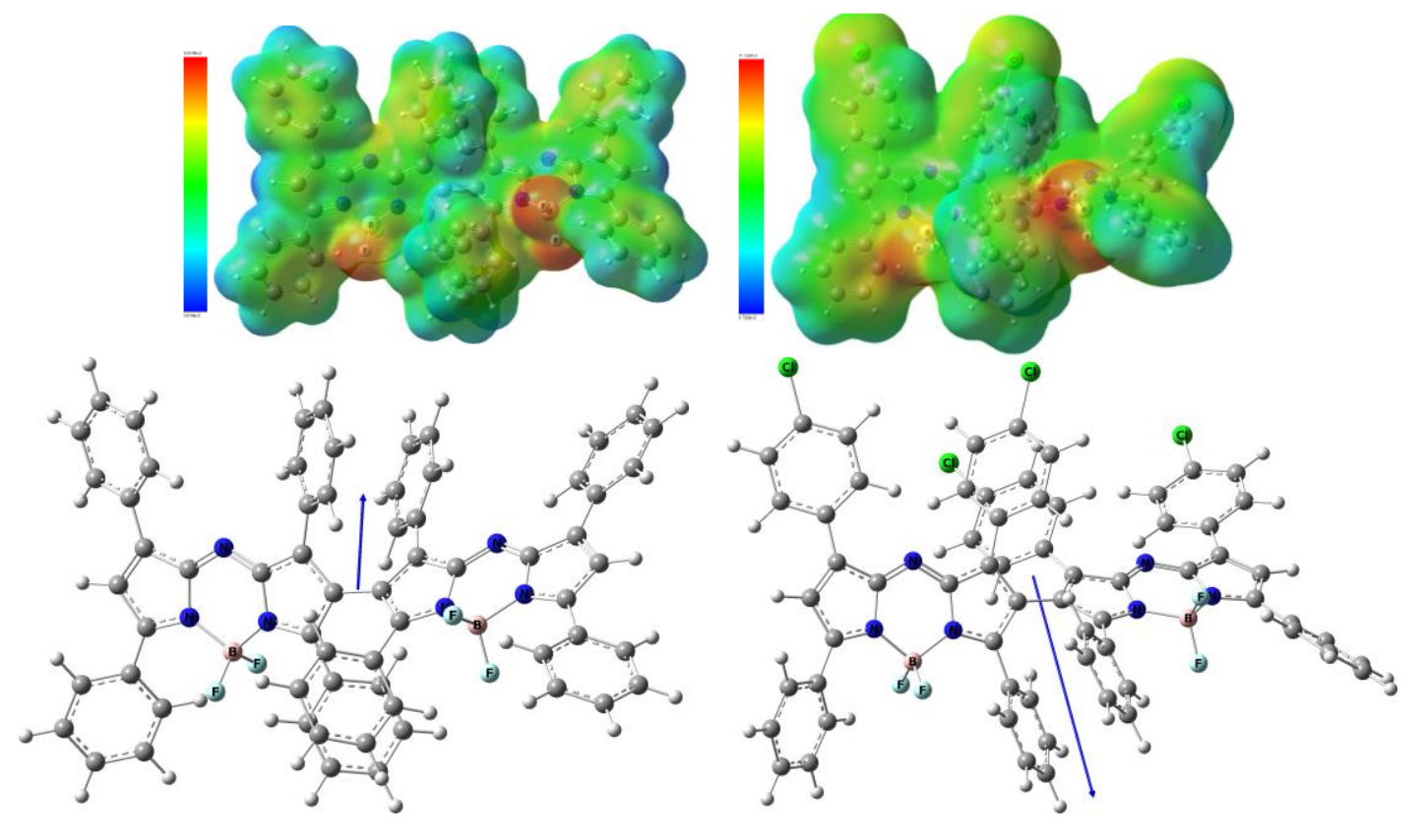


Figure 4. MEP maps (above) and dipole moment vectors (below) of dimeric aza-BODIPY and dimeric dichloro-aza-BODIPY compounds at M06-2X/cc-pVDZ level

\subsection{NBO Analysis}

NBO analysis, in which the density matrix is divided into one center and two-center orbitals demonstrating a Lewis type structure, is widely applied to shed light not only on the Lewis structure of stable molecules, but also on the reactive intermediates, namely radical, diradical, carbene, zwitterionic species, etc. [55-57]. According to the one center and two-center orbitals analyses, the occupancy reveals the following Lewis type structure indicating the electron density of 0.90 located only on p-orbital of $\mathrm{C}_{\mathrm{b}}$, and a lone-pair of 1.89 for $\mathrm{N}_{\mathrm{f}}$, found on sp2.7-orbital (Figure 5). Two empty orbitals of each 0.41 , namely sp1.2 and p orbitals, for $\mathrm{B}_{\mathrm{g}}$ form highly polarized covalent bonds with the fluorine atoms, which dedicate 1.6 electrons to each $\mathrm{B}-\mathrm{F}$ bond. These polar covalent bonds are supported by the second order perturbation theory, analysis of the Fock matrix in NBO Basis indicating the strong interactions between the electrons of fluorine atoms and empty orbitals of boron atoms with energies of 277 and $168 \mathrm{kcal} / \mathrm{mol}$. Moreover, other polarized bonds are detected between electron rich nitrogen and boron atoms. NBO analysis undoubtedly demonstrates the polarity of the $\mathrm{B}-\mathrm{N}$ bond with the larger polarization coefficient of 0.879 for the $\mathrm{N}$ hybrid and the relatively smaller one as 0.477 for $\mathrm{B}$. The lone pairs of chlorine atoms, however, moderately interact with the $\pi$-bonds of the distal phenyl units in energy of $13.6 \mathrm{kcal} / \mathrm{mol}$. The electrons on $\mathrm{C}_{\mathrm{b}}$ interact with the $\mathrm{N}_{\mathrm{d}}=\mathrm{C}_{\mathrm{e}}$ and $\mathrm{C}_{\mathrm{c}}$ $=\mathrm{C}_{\mathrm{d}}$ double bonds with energies of 76 and $40 \mathrm{kcal} / \mathrm{mol}$, respectively. NBO analysis noticeably points toward the positive charge of +0.29 on $\mathrm{C}_{\mathrm{b}}$ indicating the electron deficient center which is strongly delocalized with electron rich $\mathrm{N}_{\mathrm{f}}(-0.38)$ over the pyrrole unit.
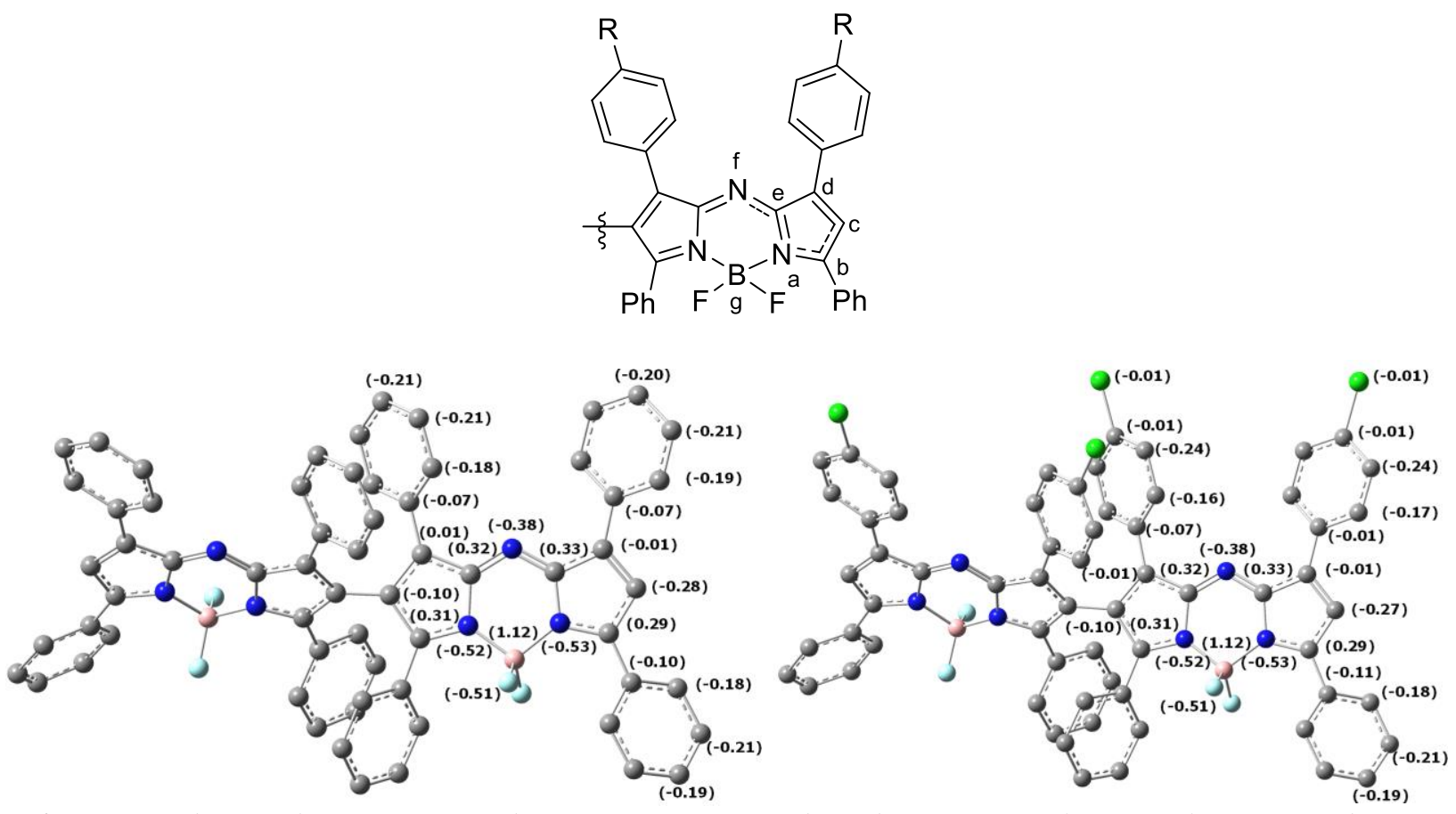

Figure 5. (Above) The structure of dimeric aza-BODIPY based on NBO analysis. (Below) NBO charges of dimeric aza-BODIPY (left) and dimeric dichloro-aza-BODIPY (right) compounds at PBEO/cc-pVTZ level

\subsection{Molecular Orbitals and Global Reactivity Properties}

The energies of FMOs are considered to assess the stability of a reaction and intramolecular charge transfer (ICT) properties of dimers, whereas the HOMO-LUMO gap was reported to be related to the stability or sensitivity of compounds [58,59]. The FMO, HOMO-1 and LUMO+1 were computed at the same level of theory, which underestimates the energies of the molecular orbitals, but provides valuable information about the understanding of the difference in molecular properties of both molecules, and their constructed surfaces are depicted in 
Figure 6. While the HOMO-1 is located on the core units and slightly spreads over the proximate phenyl units and inner distal phenyl groups with nodal planes going through the $\mathrm{BF}_{2}$ and all the nitrogen atoms in both dimers, HOMOs are mainly observed on the same atoms excluding inner distal phenyl groups. The charge density separation, resulting from the existing these nodal planes, precluded the delocalization of HOMO and HOMO-1 to the other side of the core unit. However, LUMOs and LUMO+1 are predominantly located on the core units possessing little contribution from outer distal and proximate phenyl units with nodal planes cutting the $\mathrm{BF}_{2}$ subgroups. The contribution of nitrogen orbitals is observed in these virtual orbitals. These predictions noticeably indicate the efficient ICT from the outer core units to inner core units over nitrogen atoms, which facilitate the electronic communications. Orbital composition analysis, using Mulliken, Stout \& Politzer, SCPA, Hirshfeld, Hirshfeld-I, Becke and natural atomic orbital (NAO) methods to provide the contribution of each atom to the specified molecular orbitals, can also be efficiently utilized in fully delocalized systems [60]. Orbital composition analysis renders the highest HOMO orbital compositions of $8.0-8.2 \%$ for carbon atoms connected to meso nitrogen atoms in both dimers, while the highest LUMO orbital compositions were predicted to be $8.9-9.0 \%$ for the meso nitrogen atoms (Supporting Material, S12-S14). The orbital energies show that substitution of chlorine atoms at the para positions of the distal phenyl units diminishes not only the energies of virtual orbitals from LUMO $=-2.85$ $\mathrm{eV} / \mathrm{LUMO}+1=-2.662 \mathrm{eV}$ to $\mathrm{LUMO}=-3.09 \mathrm{eV} / \mathrm{LUMO}+1=-2.90 \mathrm{eV}$, but also those of occupied orbitals from $\mathrm{HOMO}=-6.40 \mathrm{eV} / \mathrm{HOMO}-1=-6.71 \mathrm{eV}$ to $\mathrm{HOMO}=-6.60 \mathrm{eV} / \mathrm{HOMO}-1=-6.92 \mathrm{eV}$. Thus, it enhances the stability of the molecule against oxidation, but the effect is more pronounced in the virtual orbitals. As a result, the HOMO-LUMO gap of dichloro-derivative is smaller than that of the dimeric azaBODIPY molecule by $35.0 \mathrm{meV}$. $-4.90 \mathrm{eV}$ or lower energy is required for the HOMOs of molecules to achieve stable molecules against oxidation. The predicted $\mathrm{HOMO}$ energies of -6.40 and $-6.60 \mathrm{eV}$ point out the good stabilities of both compounds. HOMO energy $(-6.40 \mathrm{eV})$ of the unsubstituted dimer is slightly lower than that of the monomer $(-5.58 \mathrm{eV})(44)$, which unambiguously indicates the increase in stabilization via dimerization [45]. Although M06-2X correctly predicts the global minimum conformer and accurately estimates the lowest energy conformer [61], M06-2X/cc-pVDZ level computation overestimates the LUMO energy $(-2.85 \mathrm{eV})$ somewhat, which was calculated to be $-3.42 \mathrm{eV}$ for the monomer at B3LYP/TZVP level. The situation may emerge from the disturbance of the inner distal phenyl units in dimer. For the energy of HOMO, it can be compensated by elongation of conjugation via dimerization [62].

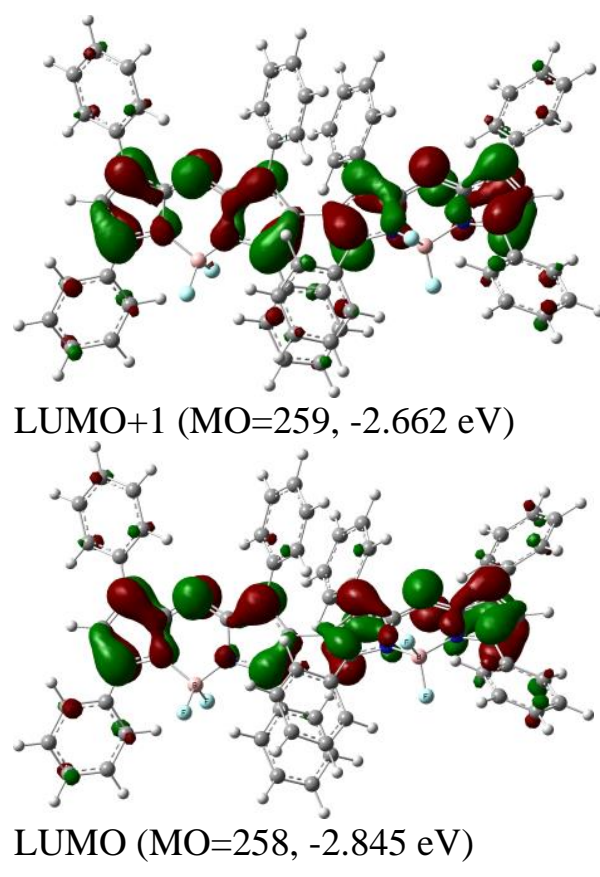

LUMO (MO=258, $-2.845 \mathrm{eV})$

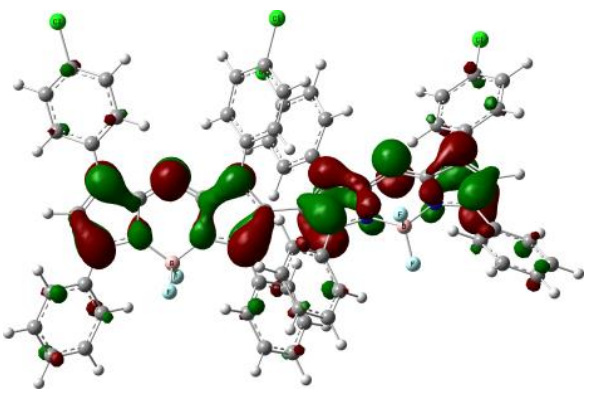

LUMO+1 (MO=291, -2.898 eV)

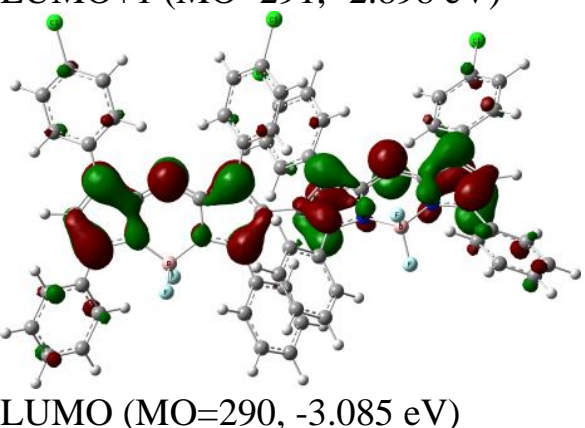



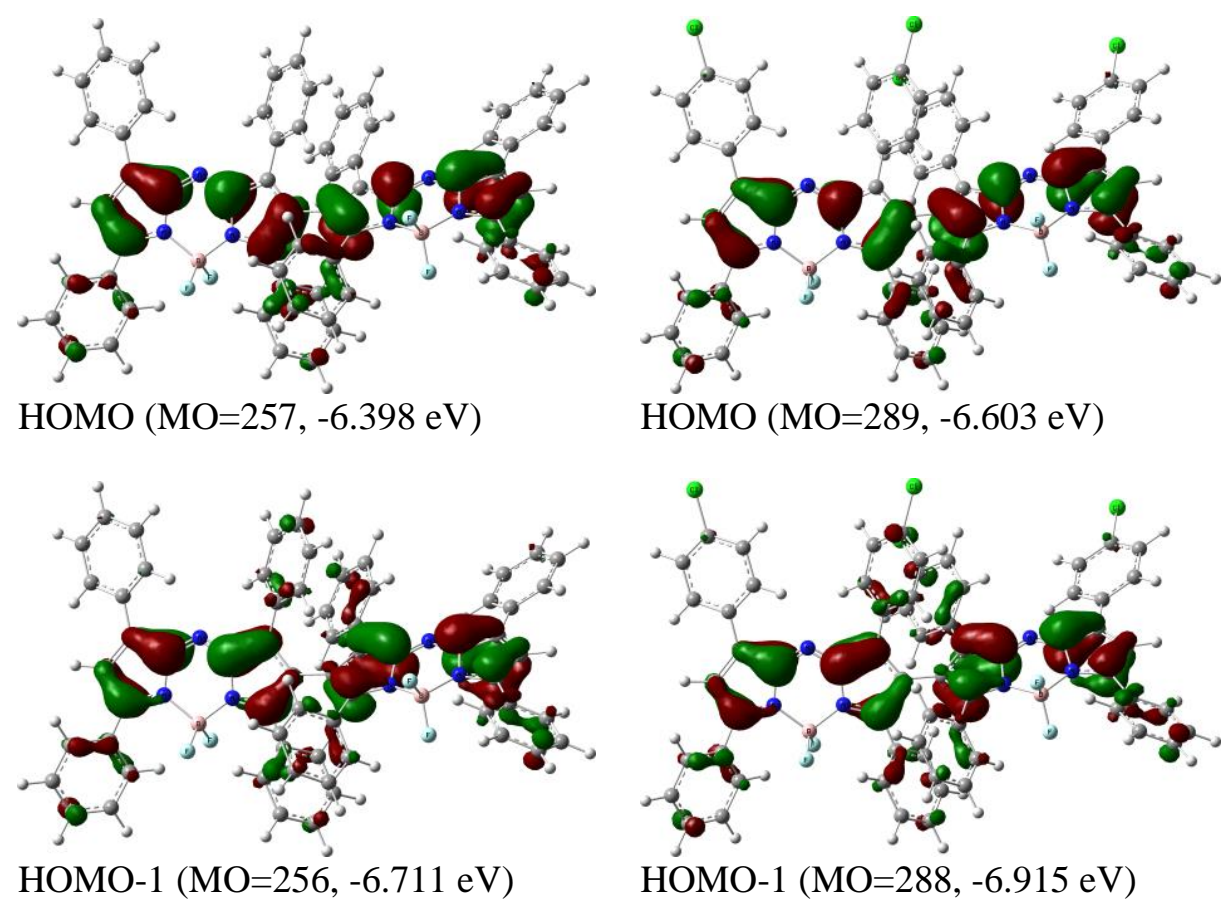

Figure 6. Molecular Orbitals (MOs) of dimeric aza-BODIPY (left) and dimeric dichloro-aza-BODIPY (right) compounds at M06-2X/cc-pVDZ level with isosurface values of 0.03 a.u $\left(E_{\text {LUмо-номо }}=3.553\right.$ and $3.518 \mathrm{eV}$, respectively)

The energies of FMOs can be utilized to derive some global reactivity properties. Ionization potential (IP $\left.=-E_{\mathrm{HOмO}}\right)$ is a physical property, the energy required to remove one or more electrons from neutral atom/molecule in the gas phase to form positively charged ions, which affects the chemical behavior of the atom/molecule $[63,64]$. The predicted IP values of the dimeric aza-BODIPY and its dichloro-derivative are 6.40 and $6.60 \mathrm{eV}$, respectively, which emphasize the higher reactivity of the dimeric aza-BODIPY molecule with respect to dichloro aza-BODIPY emerging from the mesomeric effect of chlorine atoms on stabilization of the system. Another property is the first vertical ionization (VI) which takes place rapidly from the HOMO in the time range of nuclear motion and gives rise to a highly vibrationally excited radical cation moiety following Koopman's theorem [65] and the Franck-Condon principle [66]. Subsequent reorganization of the radical cation occurs on a timescale of $10^{-12}-10^{-6} \mathrm{~s}$ [67]. For the estimation of VIE, SP computations were performed at unrestricted DFT level by removing one electron from the optimized neutral molecule, which provides a radical cation on the neutral state geometry with a positive charge and a spin of doublet. The obtained VIEs, gaps between the electronic energies of the molecules at neutral states and excited states, are calculated to be 7.08 and $7.30 \mathrm{eV}$ for the dimeric aza-BODIPY and its dichloroderivative, respectively, which points out the higher stability of dichloro-derivative compared to the unsubstituted molecule. The spin densities of $0.14-0.17$ were observed at carbon atoms connected to the meso nitrogen atoms indicating the high HOMO orbital compositions on these carbons, which was also supported by the orbital composition analysis. While ionization is related to the energy of HOMO, electron affinity $\left(E A=-E_{\text {LUMO }}\right)$ can be obtained from the energy of LUMO and it is the energy detected during the acquirement of an electron to form the corresponding anion in the gas phase [68]. The estimated EA values for the dimeric aza-BODIPY and its dichloro-derivative are 2.84 and $3.09 \mathrm{eV}$, respectively, highlighting the enhanced reactivity of dichloro-derivative as an electrophile compared to its dimeric aza-BODIPY molecule. The average value of the HOMO and LUMO energies provides the Mulliken absolute electronegativity $(\chi=(I P+E A) / 2)[57,58]$. The electronegativity of aza-BODIPY is predicted to be 4.62 $\mathrm{eV}$ and that of aza-dichloro-BODIPY is found as $4.85 \mathrm{eV}$. Based on the estimated electronegativities, dichloro aza-BODIPY molecule has the greater electron-attracting power. Moreover, the HOMO-LUMO gap also provides the chemical hardness $(\eta=(I P-E A) / 2)$ and the chemical softness $(S=1 / \eta)$, which 
describe the polarizability of an atom or a molecule [69,70]. $\eta$ values are predicted to be $1.78 \mathrm{eV}$ for the dimeric aza-BODIPY and $1.76 \mathrm{eV}$ for its dichloro derivate representing the slightly higher hardness of the dimeric aza-BODIPY molecule, that is, dichloro derivative can be ascribed as the softer chemical species [71]. Another important physical property is the chemical potential $(\mu=-\chi=-(I P+E A) / 2)$, which is a negative value of the Mulliken absolute electronegativity and calculated to be -4.62 and $-4.85 \mathrm{eV}$, respectively, pointing out the higher stability (being passive) of dichloro-derivative or higher activity (reactivity) of the dimeric aza-BODIPY molecule [72-74].

\subsection{Absorption Spectra}

The calculated UV-Vis absorption spectra and vertical excitation energies using TDA-DFT method with the incorporation of solvent effect (THF) are shown in Figure 7 and listed in Table 1. The computed spectral properties are well in alignment with the experimentally recorded absorption values. The $\lambda_{\max }$ values of the compounds arise from the HOMO-1 $\rightarrow$ LUMO+1 (83-86\%) transitions, whereas the low energy transitions arise from HOMO $\rightarrow$ LUMO (89\%). As DFT level computations overestimate the energies of LUMOs, as a result, the HOMO-LUMO gaps are relatively larger than those recorded experimentally [75]. Therefore, the first excitation energy, calculated at TDA-DFT level provides accurate estimations of the HOMO-LUMO gaps. The E predicted at the DFT level to be 3.553 and $3.518 \mathrm{eV}$, respectively, whereas TDA-DFT computations suggest the rather smaller HOMO-LUMO gaps of 1.89 and $1.87 \mathrm{eV}$, which are matching well with the reported literature values [25]. Low energy transition of the unsubstituted dimer $(\lambda=655 \mathrm{~nm})$ is smaller than that of the monomer, whose experimentally recorded HOMO $\rightarrow$ LUMO transition takes place at $643 \mathrm{~nm}$ (predicted value is $614 \mathrm{~nm}$ ) [47]. The estimated low energy transition $(664 \mathrm{~nm})$ of dichloro-derivative is well in alignment with the experimentally detected value of $702 \mathrm{~nm}[20]$.
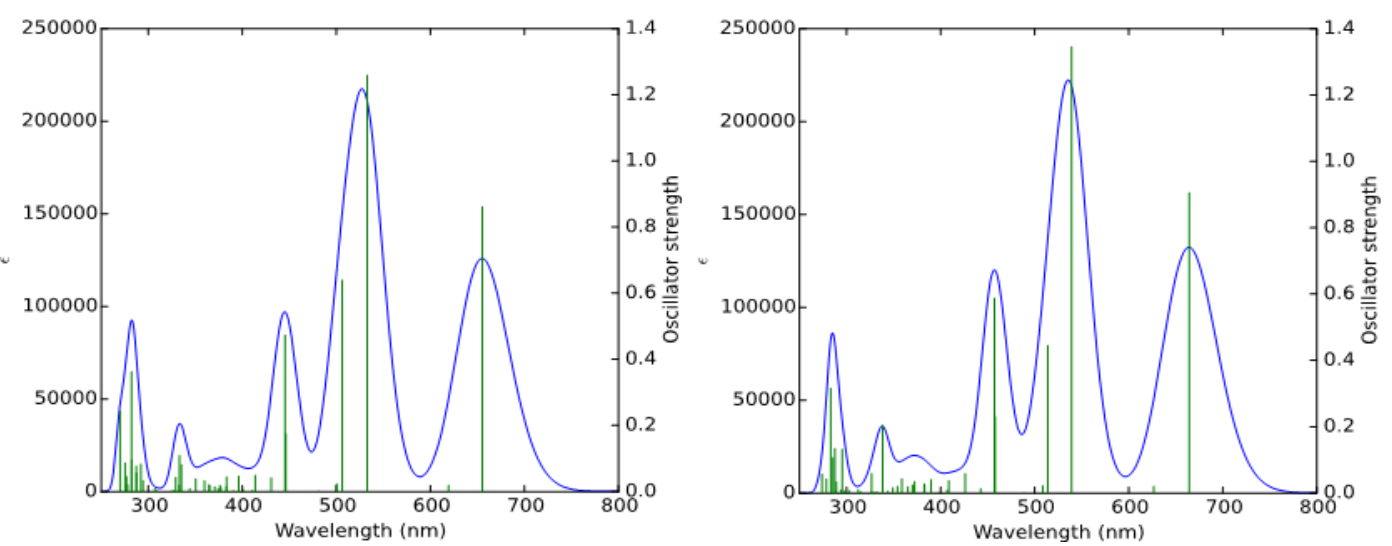

Figure 7. UV-Vis absorption spectra of dimeric aza-BODIPY (left) and dimeric dichloro-aza-BODIPY (right) compounds at (IEFPCM: THF) -TDA-PBEO/cc-pVDZ//M06-2X/cc-pVDZ level

Table 1. Excited state electronic transitions derived from (IEFPCM: THF) -TDA-PBE0/cc-pVDZ//MO6$2 X / c c-p V D Z$ level computations

\begin{tabular}{llllllll}
\hline \multicolumn{3}{c}{ Dimeric aza-BODIPY } & \multicolumn{4}{c}{ Dimeric dichloro-aza-BODIPY } \\
$\lambda_{\text {abs }}{ }^{\mathrm{a}}$ & $E^{\mathrm{b}}$ & $F^{\mathrm{c}}$ & Major contribution (\%) & $\lambda_{\text {abs }}{ }^{\mathrm{a}}$ & $E^{\mathrm{b}}$ & $F^{\mathrm{c}}$ & Major contribution (\%) \\
\hline 655 & 1.89 & 0.861 & $\mathrm{H} \rightarrow \mathrm{L}(89)$ & 664 & 1.87 & 0.906 & $\mathrm{H} \rightarrow \mathrm{L}(89)$ \\
532 & 2.33 & 1.259 & $\mathrm{H}-1 \rightarrow \mathrm{L}+1(83)$ & 539 & 2.30 & 1.346 & $\mathrm{H}-1 \rightarrow \mathrm{L}+1(86)$ \\
506 & 2.45 & 0.640 & $\mathrm{H}-2 \rightarrow \mathrm{L}(86)$ & 514 & 2.41 & 0.445 & $\mathrm{H}-2 \rightarrow \mathrm{L}(90)$ \\
445 & 2.78 & 0.473 & $\mathrm{H}-3 \rightarrow \mathrm{L}(66), \mathrm{H}-4 \rightarrow \mathrm{L}+1(25)$ & 457 & 2.71 & 0.587 & $\mathrm{H}-3 \rightarrow \mathrm{L}(67), \mathrm{H}-4 \rightarrow \mathrm{L}+1(21)$ \\
333 & 3.72 & 0.109 & $\mathrm{H}-15 \rightarrow \mathrm{L}+1(31), \mathrm{H}-16 \rightarrow \mathrm{L}(24)$ & 338 & 3.66 & 0.204 & $\mathrm{H}-12 \rightarrow \mathrm{L}+1(59), \mathrm{H}-17 \rightarrow \mathrm{L}(25)$ \\
282 & 4.39 & 0.362 & $\mathrm{H} \rightarrow \mathrm{L}+2(53), \mathrm{H}-18 \rightarrow \mathrm{L}+1(13)$ & 283 & 4.38 & 0.3216 & $\mathrm{H} \rightarrow \mathrm{L}+4(66), \mathrm{H}-1 \rightarrow \mathrm{L}+3(11)$ \\
\hline
\end{tabular}

${ }^{a}$ Wavelengths $(\lambda)$ are in nm. ${ }^{b}$ Energies (E) are given in $\mathrm{eV} .{ }^{\mathrm{c}} F$ : Oscillatory strength. (H: HOMO, L: LUMO). 


\section{CONCLUSION}

Aza-BODIPY dimers substituted by hydrogen and chlorine atoms at the para positions of the distal phenyl units were reported to demonstrate bathochromic shifts of 40 and $57 \mathrm{~nm}$ compared to their corresponding monomers, respectively. This paper has given an account of elucidation of the electronic structures, the Kohn-Sham Molecular Orbitals (MOs) and optoelectronic properties of aza-BODIPY dimers which were obtained by performing DFT computations. While orbital composition analysis renders the highest HOMO/LUMO orbital compositions in the molecules, global reactivity descriptors were utilized to predict the reactivity and stability of them. The intriguing photophysical behaviors of the dimer molecules were investigated by realizing TDA-DFT calculations rendering the low energy transitions arising from HOMO $\rightarrow$ LUMO (89\%). Further computational investigations are in progress to pave the way for new avenues of dimeric halogenated aza-BODIPYs.

\section{CONFLICTS OF INTEREST}

No conflict of interest was declared by the author.

\section{REFERENCES}

[1] Ziessel, R., Ulrich, G., Harriman, A., "The chemistry of BODIPY: A new el dorado for fluorescence tools", New Journal of Chemistry, 31: 496-501, (2007).

[2] Sevinç, G., Hayvalı, M., "The synthesis of new aryl boron-dipyrromethene compounds: Photophysical and $\mathrm{pH}$ responsive properties", Journal of the Turkish Chemical Society Section A: Chemistry, 5: 433-44, (2018).

[3] Jiao, L., Yu, C., Wang, J., Briggs, E.A., Besley, N.A., Robinson, D., Ruedas-Rama, M.J., Orte, A., Crovetto, L., Talavera, E.M., Alvarez-Pez, J.M., Van der Auweraerd, M., Boens, N., "Unusual spectroscopic and photophysical properties of meso-tert-butylBODIPY in comparison to related alkylated BODIPY dyes", RSC Advances, 5: 89375-89388, (2015).

[4] Dhokale, B., Jadhav, T., Mobin, S.M., Misra, R., "Meso enyne substituted BODIPYs: synthesis, structure and properties", Dalton Transactions, 44: 15803-15812, (2015).

[5] Zinna, F., Bruhn, T., Guido, C.A., Ahrens, J., Bröring, M., Di Bari, L., Pescitelli, G., "Circularly Polarized Luminescence from Axially Chiral BODIPY DYEmers: An Experimental and Computational Study", Chemistry - A European Journal, 22: 16089-16098, (2016).

[6] Treich, N.R., Wimpenny, J.D., Kieffer, I.A., Heiden, Z.M., "Synthesis and characterization of chiral and achiral diamines containing one or two BODIPY molecules", New Journal of Chemistry, 41: 14370-14378, (2017).

[7] Yu, C., Wu, Q., Tian, Z., Li, T., Hao, E., Jiao, L., "Synthesis and photophysical properties of meso-aryloxy linked BODIPY monomers, dimers, and trimer", Journal of Porphyrins and Phthalocyanines, 20: 475-489, (2016).

[8] Descalzo, A.B., Xu, H.-J., Shen, Z., Rurack, K., "Red/Near-infrared boron-dipyrromethene dyes as strongly emitting fluorophores", Annals of the New York Academy of Sciences, 1130: 164-171, (2008).

[9] Antina, E.V., Bumagina, N.A., "Tetraaryl-substituted aza-BODIPY: synthesis, spectral properties, and possible applications", Chemistry of Heterocyclic Compounds, 53: 39-41, (2017). 
[10] Allik, T. H., Hermes, R. E., Sathyamoorthi, G., Boyer, J. H., "Spectroscopy and laser performance of new BF2-complex dyes in solution", Proceedings of SPIE - The International Society for Optical Engineering, 2115-2129, 240, (1994).

[11] Sathyamoorthi, G., Soong, M. L., Ross, T. W., Boyer, J. H., "Fluorescent tricyclic $\beta$ azavinamidine-BF2 complexes", Heteroatom Chemistry, 4, 603, (1993).

[12] Kage, Y., Mori, S., Ide, M., Saeki, A., Furuta, H., Shimizu, S., "Blackening of aza-BODIPY analogues by simple dimerization: panchromatic absorption of a pyrrolopyrrole aza-BODIPY dimer", Materials Chemistry Frontiers, 2: 112-120, (2018).

[13] Liu, H., Lu, H., Zhou, Z., Shimizu, S., Li, Z., Kobayashi, N., Shen, Z., “Asymmetric coreexpanded aza-BODIPY analogues: facile synthesis and optical properties", Chemical Communications, 51: 1713-1716, (2015).

[14] Jiang, X.-D., Guan, J., Zhao, J., Le Guennic, B., Jacquemin, D., Zhang, Z., Chen, S., Xiao, L., "Synthesis, structure and photophysical properties of NIR aza-BODIPYs with F/N3/NH2 groups at 1,7-positions", Dyes and Pigments, 136: 619-626, (2017).

[15] Kolemen, S., Cakmak, Y., Ozdemir, T., Erten-Ela, S., Buyuktemiz, M., Dede, Y., Akkaya, E.U., "Design and characterization of BODIPY derivatives for bulk heterojunction solar cells", Tetrahedron, 70: 6229-6234, (2014).

[16] Qi, X., Kim, S.K., Han, S.J., Xu, L., Jee, A.Y., Kim, H.N., Lee, C., Kim, Y., Lee, M., Kim, S.-J., Yoon, J., "New BODIPY-triazine based tripod fluorescent systems", Tetrahedron Letters, 49: 261-264, (2008).

[17] Adarsh, N., Avirah, R.R., Ramaiah, D., "Tuning photosensitized singlet oxygen generation efficiency of novel aza-BODIPY dyes", Organic Letters, 12: 5720-5723, (2010).

[18] Wang, Y., Chen, L., El-Shishtawy, R.M., Aziz, S.G., Müllen, K., "Synthesis and optophysical properties of dimeric aza-BODIPY dyes with a push-pull benzodipyrrolidone core", Chemical Communications, 50: 11540-11542, (2014).

[19] Nepomnyashchii, A.B., Bröring, M., Ahrens, J., Bard, A. J., "Synthesis, photophysical, electrochemical, and electrogenerated chemiluminescence studies. Multiple sequential electron transfers in BODIPY monomers, dimers, trimers, and polymer", Journal of the American Chemical Society, 133: 8633-8645, (2011).

[20] Çınar, H.Ş., Özçelik, Ş., Kaya, K., Dülger Kutlu, Ö., Erdoğmuş, A., Gül, A., "Synthesis and photophysical properties of monomeric and dimeric halogenated aza-BODIPYs", Journal of Molecular Structure, 1200: 127108, (2020).

[21] Tamm, I., "Relativistic interaction of elementary particles", Russian Physics Journal, 9: 449-460, (1945).

[22] Dancoff, S.M., "Non-Adiabatic Meson Theory of Nuclear Forces", Physical Review Journals, 78: 382-385, (1950).

[23] Frisch, M.J., Trucks, G.W., Schlegel, H.B., Scuseria, G.E., Robb, M.A., Cheeseman, J.R., Scalmani, G., Barone, V., Petersson, G.A., Nakatsuji, H., Li, X., Caricato, M., Marenich, A.V., Bloino, J., Janesko, B.G., Gomperts, R., Mennucci, B., Hratchian, H.P., Ortiz, J.V., Izmaylov, A.F., Sonnenberg, J.L., Williams-Young, D., Ding, F., Lipparini, F., Egidi, F., Goings, J., Peng, B., Petrone, A., Henderson, T., Ranasinghe, D., Zakrzewski, V.G., Gao, J., Rega, N., Zheng, G., Liang, W., Hada, M., Ehara, M., Toyota, K., Fukuda, R., Hasegawa, J., Ishida, M., Nakajima, T., 
Honda, Y., Kitao, O., Nakai, H., Vreven, T., Throssell, K., Montgomery, J.A. Jr., Peralta, J.E., Ogliaro, F., Bearpark, M.J., Heyd, J.J., Brothers, E.N., Kudin, K.N., Staroverov, V.N., Keith, T.A., Kobayashi, R., Normand, J., Raghavachari, K., Rendell, A.P., Burant, J.C., Iyengar, S.S., Tomasi, J., Cossi, M., Millam, J.M., Klene, M., Adamo, C., Cammi, R., Ochterski, J.W., Martin, R.L., Morokuma, K., Farkas, O., Foresman, J.B., Fox, D.J., "Gaussian 09”, Rev.D.01, Gaussian, Inc., Wallingford CT, 2013.

[24] Zhao, Y., Truhlar, D., "The M06 suite of density functionals for main group thermochemistry, thermochemical kinetics, noncovalent interactions, excited states, and transition elements: Two new functionals and systematic testing of four M06-class functionals and 12 other functionals", Theoretical Chemistry Accounts, 120: 215-241, (2008).

[25] Momeni, M.R., Brown, A., "A local CC2 and TDA-DFT double hybrid study on BODIPY/azaBODIPY dimers as heavy atom free triplet photosensitizers for photodynamic therapy applications", The Journal of Physical Chemistry A, 120: 2550-2560, (2016).

[26] Ozmaldar, A., Cakar, Z.P., Balta, B., "The investigation of oxidative decomposition of phenyl alanine by transition metal ions", Journal of the Turkish Chemical Society Section A: Chemistry, 90-92, (2015).

[27] Grimme, S., Antony, J., Ehrlich, S., Krieg, H., "A consistent and accurate ab initio parameterization of density functional dispersion correction (DFT-D) for the 94 elements H-Pu", The Journal of Chemical Physics, 132: 154104(1-19), (2010).

[28] Grimme, S., "Density functional theory with London dispersion corrections", WIREs Computational Molecular Science, 1: 211-228, (2011).

[29] Kruse, H., Goerigk, L., Grimme, L., "Why the standard B3LYP/6-31G* model chemistry should not be used in DFT calculations of molecular thermochemistry: Understanding and correcting the problem", The Journal of Organic Chemistry, 77: 10824-10834, (2012).

[30] Goerigk, L., Grimme, S., "A thorough benchmark of density functional methods for general main group thermochemistry, kinetics, and noncovalent interactions", Physical Chemistry Chemical Physics, 13: 6670-6688, (2011).

[31] Hohenstein, E.G., Chill, S.T., Sherrill, C.D., "Assessment of the Performance of the M05-2X and M06-2X Exchange-Correlation Functionals for Noncovalent Interactions in Biomolecules", Journal of Chemical Theory and Computation, 4: 1996-2000, (2008).

[32] Lu, T., Chen, F., "A multifunctional wavefunction analyzer", Journal of Computational Chemistry, 33: 580-592, (2012).

[33] Foster, J.P., Weinhold, F., "Natural hybrid orbitals", Journal of the American Chemical Society, 102: 7211-7218, (1980).

[34] Reed, A.D., Curtiss, L.A., Weinhold, F., "Intermolecular interactions from a natural bond orbital, donor-acceptor viewpoint”, Chemical Reviews, 88: 899-926, (1988).

[35] Weinhold, F., Landis, C.R., Glendening, E.D., "What is NBO analysis and how is it useful?", International Reviews in Physical Chemistry, 35: 399-440, (2016).

[36] Glendering, E.D., Reed, A.E., Carpenter, J.E., Weinhold, F., NBO Version 3.1, Theoretical Chemistry Institute, University of Wisconsin, Madison, 1998. 
[37] Glendening, E.D., Reed, A.E., Carpenter, J.E., Weinhold, F., “NBO 3.1 Program Manual”, 1988.

[38] Grimme, S., Neese, F., "Double-hybrid density functional theory for excited electronic states of molecules", The Journal of Chemical Physics, 127: 154116, (1-18), (2007).

[39] Ernzerhof, M., Scuseria, G.E., "Assessment of the Perdew-Burke-Ernzerhof exchange-correlation functional", The Journal of Chemical Physics, 110: 5029-5036, (1999).

[40] Alberto, M.E., de Simone, B.C., Mazzone, G., Quartarolo, A.D., Russo, N., "Theoretical determination of electronic spectra and intersystem spin-orbit coupling: the case of isoindoleBODIPY dyes", Journal of Chemical Theory and Computation, 10: 4006-4013, (2014).

[41] Momeni, M.R., Brown, A., "Why do TD-DFT excitation energies of BODIPY/aza-BODIPY families largely deviate from experiment? Answers from electron correlated and multireference methods", Journal of Chemical Theory and Computation, 11: 2619-2632, (2015).

[42] Mennucci, B., Cances, E., Tomasi, J., "Evaluation of solvent effects in isotropic and anisotropic dielectrics and in ionic solutions with a unified integral equation method: Theoretical bases, computational implementation, and numerical applications", The Journal of Physical Chemistry B, 101: 10506-10517, (1997).

[43] Dennington, R., Keith, T., Millam, J., “GaussView”, Version 5, Semichem Inc.: Shawnee Mission, K.S. (2009).

[44] O'Boyle, N.M., Tenderholt, A.L., Langner, K.M., "cclib: A library for package-independent computational chemistry algorithms", Journal of Computational Chemistry, 29: 839-845, (2008).

[45] Cinar, M.E., Ozturk, T., "Thienothiophenes, dithienothiophenes, and thienoacenes: Syntheses, oligomers, polymers, and properties", Chemical Reviews, 115: 3036-3140, (2015).

[46] Killoran, J., Allen, L., Gallagher, J.F., Gallagher, W.M., O'Shea, D.F., "Synthesis of BF 2 chelates of tetraarylazadipyrromethenes and evidence for their photodynamic therapeutic behavior", Chemical Communications, 1862-1863, (2002).

[47] Zhang, X., Yu, H., Xiao, Y., "Replacing phenyl ring with thiophene: an approach to longer wavelength aza-dipyrromethene boron difluoride (aza-BODIPY) dyes", The Journal of Organic Chemistry, 77: 669-673, (2012).

[48] Jackson, N.E., Savoie, B.M., Kohlstedt, K.L., de la Cruz, M.O., Schatz, G.C., Chen, L.X., Ratner, M.A., "Controlling conformations of conjugated polymers and small molecules: the role of nonbonding interactions", Journal of the American Chemical Society, 135: 10475-10483, (2013).

[49] Gorman, A., Killoran, J., O’Shea, C., Kenna, T., Gallagher, W.M., O’Shea, D.F., "In vitro demonstration of the heavy-atom effect for photodynamic therapy", Journal of the American Chemical Society, 126: 10619-10631, (2004).

[50] Wiberg, K.B., Rablen, P.R., "A comparison of atomic charges derived via different procedures", Journal of Computational Chemistry, 14: 1504-1518, (1993).

[51] Shi, W., Liu, J., An, Z., Zhang, Q., Wang, C., "Theoretical Investigations into The Effects of Electric Field on Hydroxylamine Cation", Gazi University Journal of Science, 30 (1): 167-174, (2017). 
[52] Ersanl1, C., "Synthesis, X-ray and Quantum Chemical Characterizations Studies on (E)-2-Bromo4-chloro-6-[(4-chloro-2,5-dimethoxyphenylimino) methyl] phenol Compound", Gazi University Journal of Science, 30 (4): 531-543, (2018).

[53] Lingam, C.B., Tewari, S.P., “Theoretical studies on aminoborane oligomers”, Computational and Theoretical Chemistry, 1020: 151-156, (2013).

[54] Ekincioğlu, Y., Kılıç, H., Dereli, Ö., “A DFT/TD-DFT study on the Molecular Structure Absorption and Fluorescence Spectra of Gas/Solution Phases Adenosine 5'-triphosphate Molecule", Gazi University Journal of Science, 35: ASAP, (2021). DOI: 10.35378/gujs.834249

[55] Schmittel, M., Steffen, J.-P., Rodríguez, D., Engelen, B., Neumann, E., Cinar, M.E., "Thermal C2-C6 cyclization of enyne-carbodiimides: Experimental evidence contradicts a diradical and suggests a carbene intermediate", The Journal of Organic Chemistry, 73: 3005-3016, (2008).

[56] Rana, A., Cinar, M.E., Samanta, D., Schmittel, M., "Mechanistic information from nonstationary points", Organic Letters, 18: 84-87, (2016).

[57] Cinar, M.E., "Thermal reaction of 1,8-diketone: A computational study", Computational and Theoretical Chemistry, 1170: 112641 (1-10), (2019).

[58] Xiao, H.-M., Li, Y.-Y., "Banding and electronic structures of metal azides. Sensitivity and conductivity”, Science in China Series B Chemistry, 38: 538-545, (1995).

[59] Lingam, C.B., Babu, K.R., Tewari, S.P., Vaitheeswaran G., "Quantum chemical studies on beryllium hydride oligomers", Computational and Theoretical Chemistry, 963: 371-377, (2011).

[60] Turkoglu, G., Cinar, M.E., "Experimental and computational studies on the absorption properties of novel formazan derivatives", Turkish Journal of Chemistry, 41: 710-727, (2017).

[61] Walker, M., Harvey, A.J.A., Sen, A., Dessent, C.E.H., "Performance of M06, M06-2X, and M06HF density functionals for conformationally flexible anionic clusters: M06 functionals perform better than B3LYP for a model system with dispersion and ionic hydrogen-bonding interactions", The Journal of Physical Chemistry A, 117: 12590-12600, (2013).

[62] Pekbelgin Karaoğlu, H., Kalkan Burat, A., "Synthesis and photophysical properties of usymmetrically substituted phthalocyanine-pyrene conjugate", Journal of the Turkish Chemical Society Section A: Chemistry, 328-319, (2019).

[63] Mulliken, R.S., "A new electroaffinity scale; together with data on valence states and on valence ionization potentials and electron affinities", The Journal of Chemical Physics, 2: 782-793, (1934).

[64] Mulliken, R.S., "Electronic structures of molecules XI. Electroaffinity, molecular orbitals and dipole moments", The Journal of Chemical Physics, 3: 573-585, (1935).

[65] Koopmans, T., "Über die Zuordnung von Wellenfunktionen und Eigenwerten zu den einzelnen Elektronen eines Atoms", Physica, 1: 104-113, (1934).

[66] Franck, J., "Elementary processes of photochemical reactions", Transactions of the Faraday Society, 21: 536-542, (1926). 
[67] Shukla, R., Thakur, K., Chebny, V.J., Reid, S.A., Rathore, R., "Direct observation of electrontransfer-induced conformational transformation (molecular actuation) in a bichromophoric electron donor", The Journal of Physical Chemistry B, 114: 14592-14595, (2010).

[68] Zhan, C.-G., Nichols, J.A., Dixon, D.A., „Ionization potential, electron affinity, electronegativity, hardness, and electron excitation energy: molecular properties from density functional theory orbital energies", The Journal of Physical Chemistry A, 107: 4184-4195, (2003).

[69] Pearson, R.G., "Hard and soft acids and bases hsab.1.fundamental principles", Journal of Chemical Education, 45: 581-587, (1968).

[70] Pearson, R.G., Songstad, J., "Application of the principle of hard and soft acids and bases to organic chemistry", Journal of the American Chemical Society, 89: 1827-1836, (1967).

[71] Parr, R.G., Pearson, R.G., "Absolute hardness-companion parameter to absolute electronegativity”, Journal of the American Chemical Society, 105: 7512-7516, (1983).

[72] Parr, R.G., Von Szentpaly, L., Liu, S.B., "Electrophilicity index", Journal of the American Chemical Society, 121: 1922-1924, (1999).

[73] Pearson, R.G., "Maximum chemical and physical hardness", Journal of Chemical Education, 76: 267-275, (1999).

[74] Chattaraj, P.K., Sarkar, U., Roy, D.R., "Electrophilicity index", Chemical Reviews, 106: 2065-2091, (2006).

[75] Zhang, G., Musgrave, C.B., "Comparison of DFT methods for molecular orbital eigenvalue calculations", The Journal of Physical Chemistry A, 111: 1554-1561, (2007). 